\title{
Acute obstructive pancreatitis secondary to migration of a gastrostomy tube into duodenum
}

\author{
Waka Yanagisawa ${ }^{1}$, Daniel $\mathrm{Oh}^{2}$, Dinushi Perera ${ }^{2}$, and Sebastian Rodrigues ${ }^{2}$ \\ ${ }^{1}$ Prince of Wales Hospital and Community Health Services \\ ${ }^{2}$ Dubbo Base Hospital
}

November 13, 2021

\begin{abstract}
Percutaneous endoscopic gastrostomy (PEG) tube is a common procedure. This discusses the rare complication of acute pancreatitis, due to tube migration, causing obstruction of the ampulla of Vater. Radiological confirmation of tubes prior to usage may aid in preventing this reversible complication.
\end{abstract}

\section{Introduction}

PEG tube placements have become a common endoscopic procedure, and a preferred route for long-term enteral nutrition in patients unable to tolerate oral intake whilst maintaining gut integrity. ${ }^{1}$ They offer several advantages over nasogastric tube feeds such as better tolerance of feeds, greater caloric intake and weight gain. $^{2}$ Insertion of PEG tubes are known to be a relatively safe procedure with the more common complications described being wound infection, tube blockage, accidental removal, and gastric outlet obstruction due to tube migration. ${ }^{1}$ Migration of the tube into the duodenum, resulting in pancreatitis due to the obstruction of the ampulla of Vater, is an uncommon complication of PEG tubes which is discussed in this case. This report discusses the importance of radiological confirmation of tubes to prevent these rare complications.

\section{Case Report}

A 52-year-old female presented with a 5-day history of vomiting with epigastric pain. Her past medical history included a previous history of stroke, for which her significant dysphagia resulted in dependence on percutaneous endoscopic gastrostomy (PEG) feeds. She had previously had a laparoscopic cholecystectomy and no alcohol intake. Her PEG tube had been inserted 3 months prior with good tolerance of feeds. However, two weeks prior to this presentation, her tube had blocked at the nursing home, and replaced bedside. Unfortunately, radiological confirmation of positioning was not performed at this time prior to usage of the PEG for feeds.

On examination she was maximally tender in the epigastrium. Her bloods demonstrated a lipase of $7365 \mathrm{U} / \mathrm{L}$ (upper limit of normal being 60U/L), alkaline phosphatase 140U/L, y-glutamyl transferase 117U/L, alanine aminotransferase $98 \mathrm{U} / \mathrm{L}$, aspartate aminotransferase $78 \mathrm{U} / \mathrm{L}$ and bilirubin of $5 \mathrm{umol} / \mathrm{L}$. Her white cell count and $\mathrm{C}$ reactive protein were 15.7 and 49 , respectively. Her triglycerides, immunoglobulin G subtypes, and calcium levels were within normal ranges. Contrast enhanced computed tomography of the abdomen demonstrated a PEG tube and balloon in the region of the second part of the duodenum with associated oedema of the pancreas without any evidence of significant biliary dilatation (Figures 1 and 2). A Magnetic Resonance Cholangiopancreatography (MRCP) was performed to ensure nil other causes of ductal obstruction, and it showed evidence of uncomplicated interstitial pancreatitis without evidence of other ductal pathology (Figure 3). She had no other obvious indicators of causes for the pancreatitis other than the PEG tube balloon in the duodenum. The patient's balloon was deflated and pulled back $10 \mathrm{~cm}$ with subsequent $\mathrm{CT}$ 
imaging confirming appropriate position in the stomach. Her symptoms and inflammatory markers improved following readjustment of the PEG tube, and after a period of monitoring, she was successfully discharged.

\section{Discussion}

Gastrostomy tubes are increasingly being utilised to facilitate patients with no or minimal oral intake. Complication rates from G-tubes are known to be low, including bleeding, infection, formation of granulation tissue and tube blockage ${ }^{4}$. In particular, acute obstructive pancreatitis due to obstruction of major duodenal papilla are rare and infrequently reported in literature, and in Australian literature, none yet published. To our knowledge, there are limited reports of acute pancreatitis caused by PEG tube migration reported internationally as well ${ }^{3-5}$. Migration has been associated with Foley catheter usage, using tubes without external bumpers or not performing radiological confirmation ${ }^{6}$. Only one study performed an Endoscopic Retrograde Cholangiopancreatography (ERCP) to rule out other ductal pathology apart from the PEG tube. In our case, an MRCP was performed to ensure no other cause for pancreatitis could be determined. According to available literature, majority of known cases of PEG tube pancreatitis were due to the use of foley catheters, which have increased propensity to migrate due to external bumper dislocation. ${ }^{5}$ This case adds to the existing literature about lack of imaging following re-insertion of a gastrostomy tube as another potential contributing factor for iatrogenic pancreatitis. We have demonstrated that the appropriate management of pancreatitis secondary to PEG tube is to deflate the balloon and withdraw the tube, followed by repeating imaging to confirm appropriate positioning of the balloon. This management resulted in the patient's prompt recovery. It is still imperative that other more common causes of pancreatitis are investigated for, and the diagnosis of PEG tube migration being the cause of pancreatitis is one of exclusion.

\section{Conclusion}

This is the first published case of pancreatitis secondary to PEG tube migration in Australian literature. Although this is an uncommon complication of PEG tubes, obstructive pancreatitis due to PEG tube migration have previously been described, and therefore should be considered as a potential cause in a patient with a PEG tube. This also raises the question of whether there is a role for radiological confirmation of feeding gastric tubes prior to usage to help prevent such complications. We know that PEG tubes generally have a balloon or bumper at the tip to prevent outward migration, and an external bumper to reduce the risk of inward migration. Other considerations include the use of a correct tube of appropriate length (perhaps with external markings to help guide the depth of insertion), and routinely checking that the external bumper is fit snug to the patient to minimise migration. ${ }^{5}$

\section{Conflict of interest}

None declared

\section{Ethical approval}

This study does not require any ethical committee approval.

\section{Author contributions}

WY, DO, DP and SR were all involved in reviewing the literature, preparing and editing the manuscript and all authors approved the final version of the manuscript.

\section{Data availability statement}

Data sharing is not application to this article as no new data were created or analysed in this study.

\section{References}

1. Rahnemai-Azar A, Rahnemaiazar A, Naghshizadian R, Kurtz A, Farkas DT. Percutaneous endoscopic gastrostomy: Indications, technique, complications and management. World Journal of Gastroenterology. 2014;20(24):7739-7751. 
2. Park RH, Allison MC, Lang J, Spence E, Morris AJ, Danesh BJ, Russell RI, Mills PR. Randomised comparison of percutaneous endoscopic gastrostomy and nasogastric tube feeding in patients with persisting neurological dysphagia. BMJ (Clinical research ed.) . 1992;304 (6839):1406-1409.

3. Miele VJ, Nigam A. Obstructive jaundice and pancreatitis secondary to percutaneous endoscopic gastrostomy tube migration. J Gastroenterol Hepatol. 2005;20(11):1802-1803.

4. Taylor DF, Cho R, Cho A, Nguyen V, Sunnapwar A, Womeldorph C. Obstructive acute pancreatitis secondary to PEG tube migration. ACG Case Reports Journal. 2016;3(4):e150.

5. Imamura H, Konagaya T, Hashimoto T, Kasugai K. Acute pancreatitis and cholangitis: A complication caused by a migrated gastrostomy tube. World Journal of Gastroenterology. 2007;13(39):5285-5287.

6. Bhargava, A., Andrews, C., Belforti, R. (2011). Acute pancreatitis from gastrostomy tube migration in a nursing home resident, Annals of Long-Term Care, 19 (3),

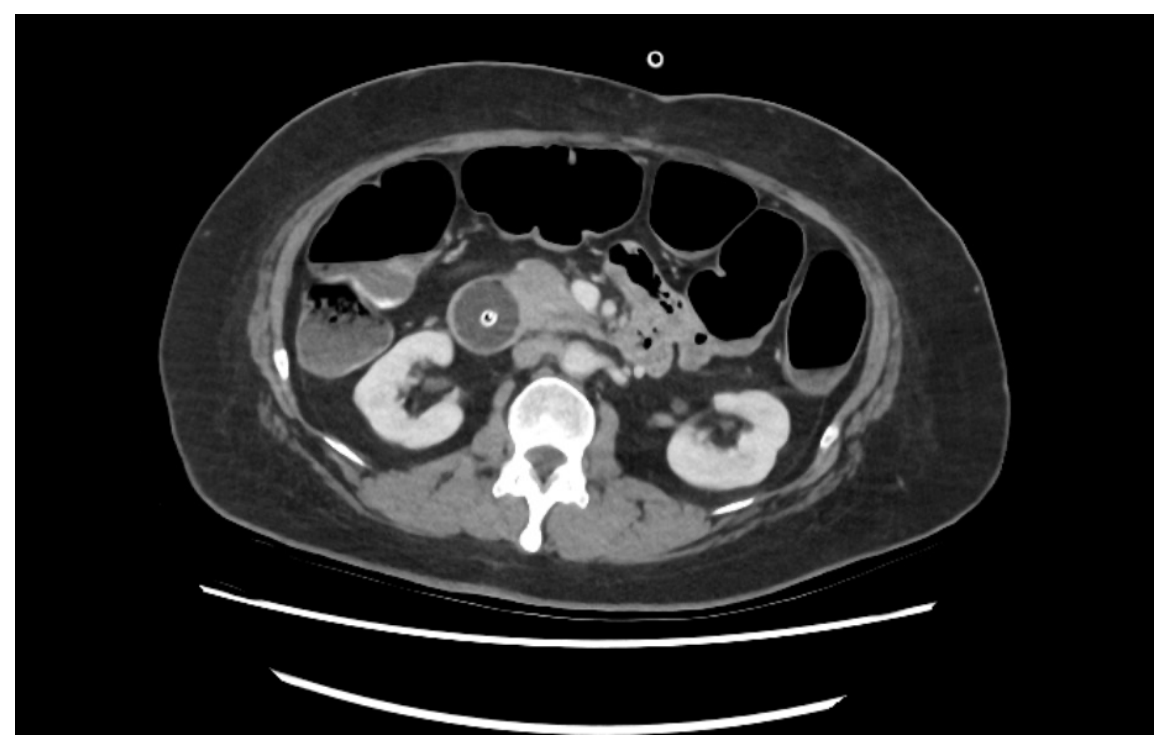




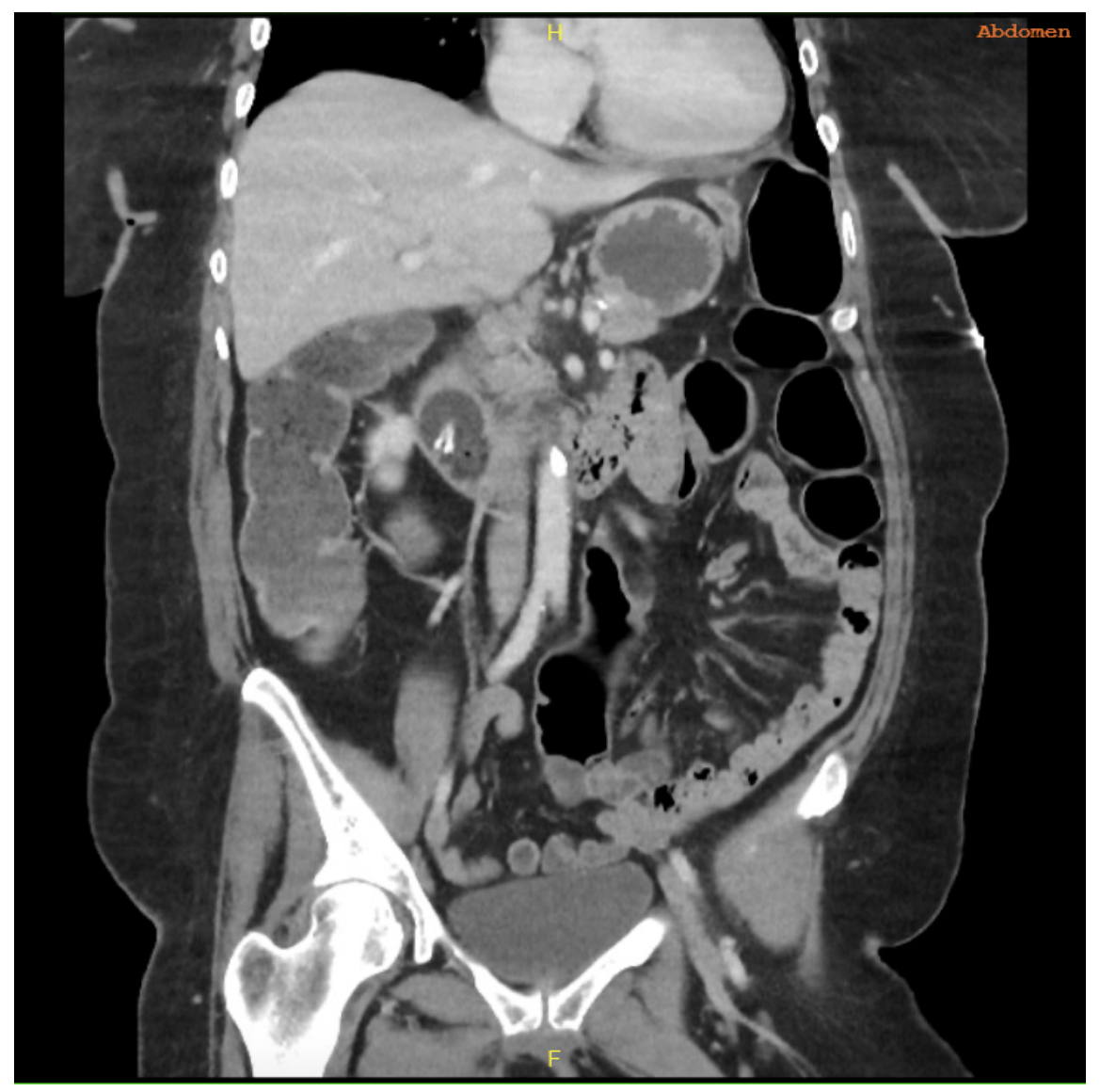




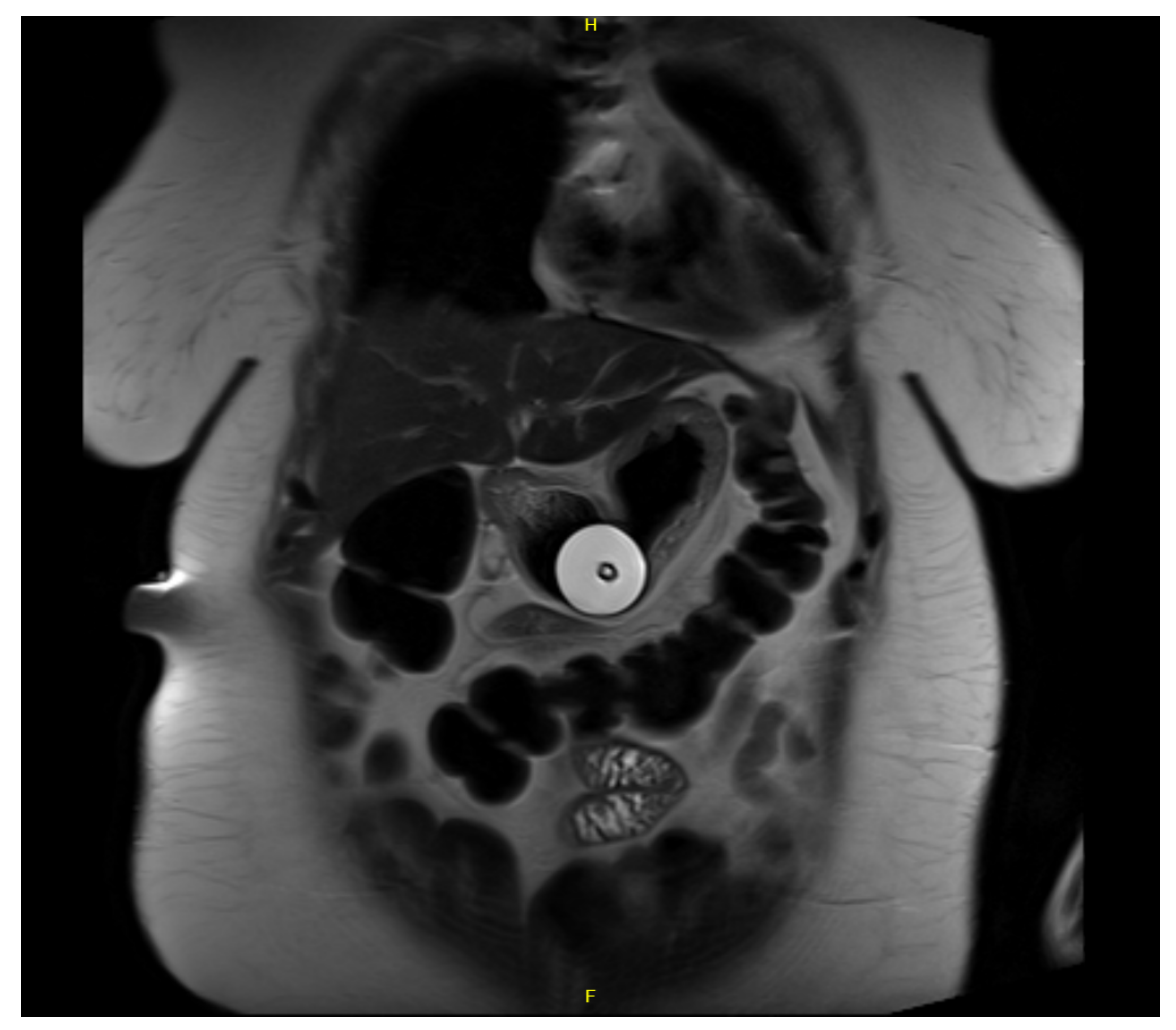

